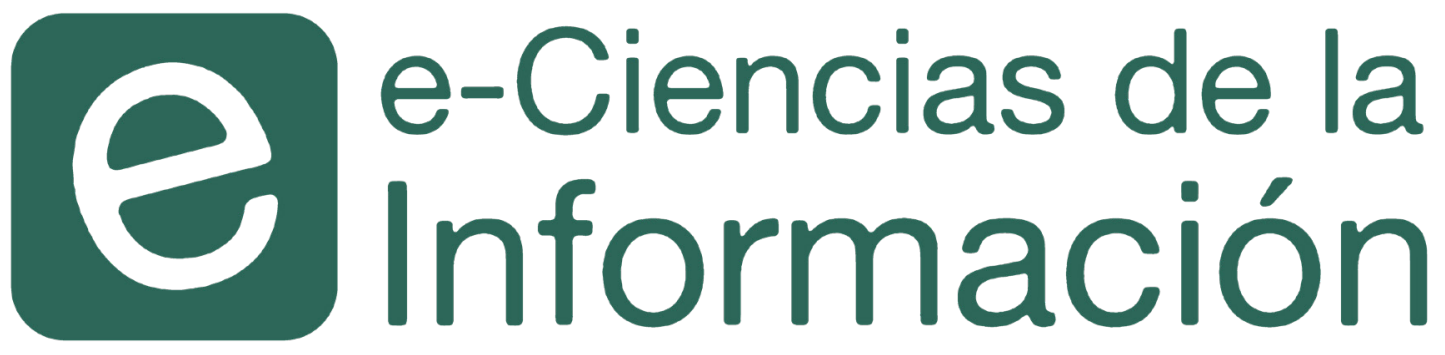

\title{
Gestión de revistas a través de OJS: Experiencia de éxito Angolana
}

\author{
Wileidys Artigas \\ Eurico Wongo Gungula
}

\section{Recibido: 8/04/2019 | Corregido: 3/09/2019 | Aceptado: 27/09/2019} DOI: 10.15517/eci.v10i1.39771

e-Ciencias de la Información, volumen 10, número 1, Ene- Jun 2020

ISSN: 1649-4142

\section{다(1)\$()}

\section{¿Cómo citar este artículo?}

Artigas, W. y Gungula, E.W. (2020). Gestión de revistas a través de OJS: Experiencia de éxito Angolana. e-Ciencias de la Información, 10(1). doi: 10.15517/eci.v10i1.39771 


\title{
Gestión de revistas a través de OJS: Experiencia de éxito Angolana
}

\author{
Gestão de revistas através do OJS: Experiência de \\ Sucesso em Angola \\ Journal Management Through OJS: Angolan Success \\ Experience \\ Wileidys Artigas (iD \\ Eurico Wongo Gungula iD $^{2}$
}

\section{RESUMEN}

El objetivo del presente artículo es describir la experiencia de la implementación del Open Journal System (OJS) como sistema de gestión de la revista Sapientiae de la Universidad Óscar Ribas (UÓR) en Luanda, Angola. Su implementación fue efectuada en el 2017 junto con una serie de acciones conducentes al manejo adecuado del OJS por parte de la dirección, de los docentes y de los investigadores de la UÓR. El enfoque metodológico seguido es de tipo descriptivo-documental, basado en pruebas documentales durante la implementación del OJS. Los resultados obtenidos evidencian la pertinencia de la implementación del OJS en las distintas Instituciones de Educación Superior angolanas, como herramienta indispensable para el perfeccionamiento del proceso de formación profesional, de publicación de resultados investigativos y de internacionalización de la labor que se realiza en los dominios científicos y tecnológicos.

Palabras Clave: Open Journal System, Sapientiae, Universidad Óscar Ribas, Angola, revistas científicas.

\section{RESUMO}

O objetivo do presente artigo é descrever a experiência da implementação do Open Journal System (OJS) como sistema de gestão da revista Sapientiae da Universidade Óscar Ribas (UÓR) em Luanda, Angola. A sua implementação foi efectuada em 2017, com uma série de ações conducentes ao manejo adequado do OJS por parte da direção, dos docentes e dos investigadores da UÓR. O enfoque metodológico seguido é de tipo descritivo-documental, baseado em provas documentais durante a implementação do OJS. Os resultados obtidos evidenciam a pertinência da implementação do OJS nas distintas Instituciones de Educación Superior angolanas, como ferramenta indispensável para o aperfeiçoamento do processo de formação profissional, de publicação de resultados investigativos e de internacionalização da atividade laboral que se realiza nos domínios científicos e tecnológicos.

1 Universidad del Zulia. VENEZUELA / Universidad Óscar Ribas. ANGOLA Correo electrónico: wileidys@ hotmail.com; ORCID: https://orcid.org/0000-0001-6169-5297

2 Universidad Óscar Ribas. ANGOLA. Correo electrónico: euricowongowongo@gmail.com. ORCID: https:// orcid.org/0000-0002-5685-1328 
Palavras Chave: Open Journal System, Sapientiae, Universidade Óscar Ribas, Angola, revistas científicas.

\section{Abstract}

The objective of this article is to describe the systematized experience in the implementation of the Open Journal System (OJS) as a management system for the Sapientiae journal of the Universidade Óscar Ribas (UÓR) in Luanda, Angola. Its implementation was in 2017 with some other actions to the proper management of the OJS by the management, the teachers and the researchers of the UÓR. The methodological approach followed is descriptive-documentary, based on documentary evidence during the implementation of the OJS. The results obtained in the UÓR demonstrate the relevance of the widespread implementation of OJS in the different Angolan Higher Education Institutions, as an indispensable tool for improving the training process, publication of research results and internationalization of the work carried out in the scientific and technological codes.

Key Words: Open Journal System, Sapientiae, Óscar Ribas University, Angola, Scientific journal.

\section{Introducción}

El acceso abierto a la información científica ha sido un debate de amplia discusión en la comunidad científica internacional. Los estudios desarrollados por Claudio-González y Villarroya (2015), a través de una encuesta aplicada a 1280 editores de revistas científicas en España, han permitido identificar tres elementos que viabilizan la implementación de estrategias de acceso abierto: a) Mejora la visibilidad, vinculada a la internacionalización de las revistas; b) Favorece y agiliza el acceso a la ciencia, y aumenta las citas (contribuyendo con el Índice) y c) Ayuda al aumento de lectores, visitas y descargas.

En el contexto angolano, se visualiza actualmente el interés de gestores de Instituciones de Educación Superior (IES) en realizar debates técnicocientíficos que contribuyan a profundizar la metodología de implementación de sistemas abiertos de publicación de resultados científicos. Cabe resaltar que el Sistema de Educación Superior Angolano es un sistema relativamente nuevo en función de aplicación de políticas de investigación,

Este articulo forma parte de un proyecto registrado en el año 2017 ante el Consejo de Desarrollo Científico y Tecnológico de la Universidad del Zulia, Venezuela (CONDES-LUZ). 
por tanto, a la fecha no cuenta con estadísticas nacionales acerca del desempeño del mismo, especialmente de los resultados de impacto internacional, siendo este un gran reto para la transformación cualitativa de la situación actual, a través de la difusión científica en revistas de prestigio nacional e internacional.

Este interés es extensivo a los gestores de distintos Centros de Estudios e Investigación Científica (CEIC), de Centros de Desarrollo e Investigación Tecnológica (CDIT), del Ministerio de Educación Superior, Ciencia Tecnología e Innovación, (MESCTI), entre otros actores sociales (Ministerio de Ciencia y Tecnologia , 2013; Faustino, Pérez Sánchez y Gungula Wongo, 2017). Esto, debido a la problemática que actualmente enfrentan, vinculada con la insuficiente visibilidad nacional e internacional de las investigaciones llevadas a cabo por las distintas IES, y el insuficiente conocimiento y experiencia que tienen en cuanto a sistemas modernos de publicación de resultados científicos, constituido como uno de los retos impuestos por el acelerado desarrollo científico y tecnológico que ocurre en el mundo.

Las motivaciones en contribuir a solucionar la problemática identificada a través de la implementación del Open Journal System (OJS), se fundamentan en las potencialidades que este sistema presenta; a fin de ajustar cada vez más, las IES angolanas a los parámetros internacionalmente recomendados para la divulgación de resultados de investigaciones teóricas o prácticas.

En aras de contribuir a superar las insuficiencias diagnosticadas en las distintas IES públicas y privadas angolanas, se describe la experiencia en la implementación del OJS como sistema de gestión de la revista Sapientiae de la UÓR en Luanda, Angola. En el artículo se presenta una contextualización del entorno universitario en Luanda con respecto a la investigación y visibilidad de las revistas científicas; seguido de las experiencias en la UÓR a través de la revista Sapientiae; finalizando con los retos que enfrenta el Ministerio de Educación Superior, Ciencia, Tecnología e Innovación de Angola ante los avances de la ciencia, tecnología e innovación.

\section{Referente contextual sobre Revistas Científicas en}

Luanda

Angola es un país en pleno proceso de reconstrucción, de hecho, como señala Borrero (2017) se conquista la paz en abril del 2002, desde entonces la comunidad internacional ha sido testigo del crecimiento impetuoso y los pasos significativos que ha dado en los últimos 15 años. Estos procesos no dejan de lado el ámbito educativo, ya que, según el Ministerio de Educación Superior, Ciencia, Tecnología e Innovación (2017), se están haciendo todos los esfuerzos por mejorar la docencia, investigación y extensión universitaria.

En este sentido, se han realizado diferentes reuniones y encuentros nacionales, en donde integrantes del sector público y privado han puesto de manifiesto la necesidad de iniciar los procesos de armonización curricular, para crear una línea común de desarrollo en el sector, (Ministerio de Educación Superior, Ciencia, Tecnología e Innovación, 2017). Con el objeto de contextualizar las acciones implementadas para la gestión de una revista científica en una IES privada en Luanda, Angola; se destaca la pertinencia de ubicar al lector en el referido contexto. 
En primera instancia, se realizó una revisión en el portal Angola Formativa ${ }^{4}$, (Angola formativa, 2018), donde se obtuvieron 19 IES privadas en la ciudad de Luanda (aparecen siete instituciones más, pero son centros de formación no universitarios), dichas IES fueron localizadas a través de sus portales web (ver tabla 1). En ese proceso se ha podido observar que cuatro de ellas poseen páginas referidas en el portal señalado, pero las mismas no ingresan en la web (ver tabla 2).

TABLA 1

IES privadas de la ciudad de Luanda, Angola (2018)

\begin{tabular}{|c|c|c|}
\hline IES & SIGLAS & ENLACE WEB \\
\hline Instituto Superior de Angola & ISA & http://www.is-angola.com \\
\hline $\begin{array}{l}\text { Instituto Superior Internacional } \\
\text { Politécnico de Angola }\end{array}$ & ISIA & https://isia.co.ao/ \\
\hline $\begin{array}{l}\text { Instituto Superior Politécnico } \\
\text { Arborecer de la Juventud }\end{array}$ & ISPAJ & http://www.ispaj.net/ \\
\hline $\begin{array}{l}\text { Instituto Superior Politécnico } \\
\text { Atlantida }\end{array}$ & ISPA & www.ispatlantida.co.ao/ \\
\hline $\begin{array}{c}\text { Instituto Superior Politécnico } \\
\text { Cazenga }\end{array}$ & ISPOCA & http://www.ispoca.com \\
\hline $\begin{array}{l}\text { Instituto Superior Politécnico de } \\
\text { Tecnologías y Ciencias }\end{array}$ & ISPTEC & http://www.isptec.co.ao/pt \\
\hline $\begin{array}{l}\text { Instituto Superior Politécnico } \\
\text { Katangoji }\end{array}$ & ISPK & http://www.ispk.co.ao/ \\
\hline $\begin{array}{l}\text { Instituto Superior Politécnico } \\
\text { Metropolitano de Angola }\end{array}$ & IMETRO & http://www.imetroangola.com/ \\
\hline $\begin{array}{c}\text { Instituto Superior Técnico de } \\
\text { Angola }\end{array}$ & ISTA & http://www.ista-angola.com \\
\hline Universidad Católica de Angola & UCAN & http://www.ucan.edu \\
\hline Universidad de Belas & UNIBELAS & http://www.unibelas.org/unibelas_site/ \\
\hline Universidad Gregorio Semedo & UGS & http://www.ugs.ed.ao \\
\hline $\begin{array}{l}\text { Universidad Independiente de } \\
\text { Angola }\end{array}$ & UNIA & http://www.unia.ao \\
\hline $\begin{array}{l}\text { Universidad Jean Piaget de } \\
\text { Angola }\end{array}$ & UNIPIAGET & http://www.unipiaget-angola.org/ \\
\hline Universidad Lusiada de Angola & ULA & http://portal.ulangola.ed.ao/ \\
\hline Universidad Metodista de Angola & UMA & http://www.uma.co.ao \\
\hline Universidad Oscar Ribas & UÓR & http://www.uor.ed.ao \\
\hline Universidad Privada de Angola & UPRA & http://www.upra.ao/\# \\
\hline Universidad Técnica de Angola & UTANGA & http://www.utanga.co.ao/ \\
\hline
\end{tabular}

Fuente: elaboración propia con base en Angola Formativa 
TABLA 2

IES sin enlace web disponible (agosto 2018)

\begin{tabular}{lc}
\multicolumn{1}{c}{ IES } & SIGLAS \\
\hline Universidad Privada de Angola & UPRA \\
Instituto Superior Politécnico Katangoji & ISPK \\
Instituto Superior Politécnico Cazenga & ISPOCA \\
Instituto Superior de Angola & ISA \\
\hline
\end{tabular}

Fuente: elaboración propia

Luego de su ubicación, se realizó la siguiente revisión en los portales disponibles:

1. Si la IES posee en su página de inicio un acceso a algún elemento relacionado a la investigación, este puede ser directamente la palabra "investigación", o también "centros de investigación", "producción científica", entre otras. En este contexto, se colocó en la tabla 3 "sí", cuando estuviese reflejado y no cuando no pudiera ubicarse en el portal.

2. Si la IES posee revista científica electrónica o alguna publicación relacionada en la web; se colocó en la tabla 3 "sí", cuando se encontraba, y no en caso contrario.

Del mismo modo, se anotan las observaciones para aquellas que resulten con respuestas positivas en cualquiera de los dos casos.

TABLA 3

Ubicación de investigación y revistas en sus portales universitarios

\begin{tabular}{lcc}
\hline INSTITUCIÓN & ENLACEA & REVISTA CIENTÍFICA \\
\hline INVESTIGACIÓN & Si $\left(^{*}\right)$ \\
ISPAJ & Sí & No \\
ISIA & No & No \\
ISPA & No & No \\
ISPTEC & Sí & Sí $(4)$ \\
IMETRO & Sí & No \\
ISTA & No & No \\
UCAN & Sí & No \\
UNIBELAS & No & No \\
UGS & Sí & No \\
UNIA & No & Sí $\left.{ }^{*}\right)$ \\
UNIPIAGET & No & No \\
ULA & No & Sí $(*)$ \\
UMA & Sí & Sí \\
UÓR & Sí & No \\
UTANGA & No & \\
\hline
\end{tabular}

(*) enlaces encontrados pero vacíos; (\#) cantidad de revistas.

Fuente: elaboración propia 
Para señalar más detalles sobre los hallazgos encontrados, se describirán aquellas que obtuvieron alguna respuesta positiva:

a) En el caso del ISPAJ se encontraron enlaces de investigación a través del enlace Unidades Orgánicas en donde incluye el Centro de Investigación y Desarrollo (CIED). En dicho portal se encontró, además, un enlace a una revista científica, sin embargo, se coloca con asterisco en función de que no hay ningún contenido en ese enlace.

b) EI ISPTEC presenta su enlace a investigación a través de la palabra "producción científica", donde señala estos enlaces: publicaciones en revistas con factor de impacto, publicaciones en revista sin factor de impacto, actas de congreso, estudios desarrollados por docentes, artículos en websites y libros; sin embargo, ninguno lleva a ninguna página adicional (solo aparece como estructura web).

c) El IMETRO señala un enlace de investigación que lleva al Centro de Estudios de Investigación Científica (CEIC), el cual se encuentra en una página desligada de la institucional y luego dentro de investigación, tiene "Ciencia y Tecnología", que lleva a la página www.ciencia.ao, que es un repositorio de documentos científicos coordinado por el Ministerio de Educación Superior, Ciencia, Tecnología e Innovación de Angola.

Dentro del CEIC se incluye un enlace a publicaciones en donde presenta cuatro publicaciones sin ISSN (International Standard Serial Number), con las siguientes características:

- $\quad$ La primera de ellas, denominada Barómetro de Coyuntura Económica, señalando que es una publicación trimestral, pero solo cumplió este enunciado en el año 2014. El último número es de 2016 (sin mes, solo segunda edición de 2016) y en el mismo aparecen solo dos documentos que parecen artículos científicos, sin embargo no resaltan el autor del artículo, que aparece como pie de página, y no cumple las características de un artículo.

- $\quad$ La segunda de ellas se denomina Observatorio de Inflación, señalando que es una publicación semanal, pero el último número salió en febrero de 2016 con solo cuatro páginas de observaciones, sin artículos.

- La tercera se denomina Observatorio de Petróleo, indicando que es mensual, y la última data de febrero de 2016, con solo cuatro páginas de observaciones, sin artículos.

- La cuarta se denomina Sondeo del Consumidor, que señala como mensual, y tiene su última edición de diciembre de 2017 con solo cuatro páginas de observaciones, sin artículos.

Las últimas tres publicaciones parecen ser boletines informativos y no revistas científicas, la que se acerca más a revista científica es la primera de ellas, aunque tampoco posee ISSN ni cumple con alguno de los estándares generales de periodicidad, cantidad de artículos, entre otros.

d) Por su lado, la UCAN presenta dentro de Unidades Orgánicas a los centros de investigación, a saber: Centro de Investigación de Derecho (CID), Laboratorio

de Ciencias Sociales y Humanidades y el Centro de Estudios e Investigación Científica (CEIC). 
e) La UGS tiene enlace con centros autónomos, en donde se encuentra el Centro de Estudios, Investigación y Desarrollo (CEID). No presenta ninguna revista electrónica.

f) En cuanto a la UNIPIAGET señala un enlace a publicaciones, pero sin contenido alguno.

g) En relación a la UMA presenta un enlace a investigación, y en relación a la revista aparece un enlace de revista científica, pero vacío.

h) Para finalizar, en cuanto a la UÓR presenta un enlace a investigación, pero no señala sus centros de investigación, dentro del mismo se encuentra la revista científica Sapientiae, dicha revista fue creada en el 2015 y se encuentra activa y actualizada.

De esta manera solo quedan dos IES con publicaciones en su web, en este caso IMETRO y UÓR, de las cuales se detallaron anteriormente sus características. Podríamos tener la duda sobre la existencia de publicaciones en el ámbito de IES públicas en Luanda, sin embargo, ampliando la revisión dentro del mismo portal, se encontraron siete IES públicas, de las cuales dos institutos funcionan dentro de la Universidad Agostino Neto (UAN) y uno de ellos es un Centro de Formación. En este sentido, se señalan tres: El Instituto Superior de Ciencias de la educación (ISCED: http://isced.ed.ao), la Universidad Agostino Neto (UAN: https://www.uan.ao/) y el Instituto Superior para Tecnologías de Información y Comunicación (ISUTIC: https://www.isutic.gov.ao/), en los cuales se observaron los enlaces a investigación, pero en ninguno apareció la existencia (por lo menos en la página web) de una revista científica. Es necesario aclarar que en la página web aparecía el Instituto Superior Internacional Politécnico de Angola (ISIA) como IES pública, sin embargo se constató en su enlace web y la misma es una IES privada.

Ampliando la búsqueda en Google, colocando la palabra Revista Científica Angola, aparecen la Revista Angolana de Sociología (publicada solo en 2011) https://revistangolanasociologia.wordpress.com/publicacoes/ y la Revista Mulemba/Revista Angolana de Ciencias Sociales (publicada hasta 2015) https://journals.openedition.org/mulemba/. Cabe destacar que estas revistas no se encuentran afiliadas a ninguna de las instituciones antes referidas, solo se amplió la búsqueda en función de descartar enlaces distintos a los oficiales de las universidades.

Según Guambe y Bueno de La Fuente (2013) también existe otra revista de acceso abierto en Luanda, Angola, denominada Revista Agromeio, sin embargo, al ingresar al enlace https://agromeio.webnode.com.br/atual/ solo presenta un número del año 2011.

Incluso en el 2013, Guambe y Bueno de La Fuente (2013) ya señalaban la escasez de publicaciones científicas digitales y en especial las de acceso abierto en la África de habla lusófona, aclarando que limita la visibilidad y difusión global de las investigaciones realizadas en dichos países.

Se puede observar entonces, cómo el desarrollo de una revista científica en Angola y en específico, dentro de la ciudad de Luanda, puede tomarse como un paso más en la búsqueda del logro del Programa de Desarrollo Nacional (2018-2022). En este sentido, el Ministerio de Educación Superior, Ciencia, Tecnología e Innovación señaló a través de la Agencia Angola Press 
(2018) que el Decreto Presidencial número 201/11 de 20 de Julio establece la implantación de los indicadores de Ciencia, Tecnología e Innovación como forma de asegurar el acompañamiento de esta actividad por el Ejecutivo, teniendo en cuenta la inversión realizada, la producción científica, tecnológica y la integración de los resultados obtenidos en la sociedad.

De esta manera, se observa el interés desde los organismos gubernamentales por incrementar la calidad de la ciencia, tecnología e innovación, siendo una de las formas de mostrar la productividad, la creación y mantenimiento de revistas científicas con el cumplimiento de los estándares internacionales, de manera que puedan servir de referencia de la investigación científica realizada en Angola (Régimen Jurídico de Evaluación y Acreditación de Calidad de las Instituciones de Educación Superior, 2018).

\section{Internacionalización de la revista Sapientiae: Experiencia de éxito en la Universidad Óscar Ribas en Luanda, Angola}

El OJS es una aplicación de código abierto que es desarrollado por el Public Knowledge Project (PKP) de Canadá , que está dedicado al aprovechamiento y desarrollo de las nuevas tecnologías para el uso en investigación académica. EI PKP trabaja a través de sus esfuerzos, financiados con fondos federales, con el fin de expandir y mejorar el acceso a la investigación (Public Knowledge Project, 2017). Según Willinsky, Stranack, Smecher, Macgregor y Acevedo (2010) el OJS cuenta con un conjunto de características que determinan su funcionalidad, pero una de las que se debe resaltar tiene que ver con la posibilidad de que cada revista configure los requisitos, secciones, pasos de revisión, entre otros, según sus necesidades.

Actualmente existen innumerables casos de éxito que pueden encontrarse en la web a través de la implementación del OJS como sistema de gestión de revistas científicas, donde a modo de ejemplo se destaca el caso de la Universidad de Murcia (Hernández Pina y Maquilón, 2010), que -como en cualquier caso de adaptación- encontraron limitaciones informáticas en el inicio del proyecto, pero luego fueron solventadas debido al gran compromiso de la Universidad de Murcia por la mejora del proceso. Siendo un sistema usado mundialmente, se puede observar su desempeño a través de los informes anuales proporcionados por PKP ${ }^{5}$.

Desde antes de la creación de la revista ya se observaba el interés del continente africano de superar estándares (Scientific Electronic Library Online [Scielo], 2014), cuando advertía sobre el interés futuro que desde África se vislumbraba sobre el tema de la investigación científica; en dicho estudio se hizo referencia a cada uno de los continentes, pero no se obtuvo información detallada por país.

La revista Sapientiae se creó en 2015 y es una revista científica arbitrada semestral de la Universidad Óscar Ribas en Luanda-Angola, comprometida con el desarrollo de la ciencia, especialmente en la difusión del conocimiento generado a través de las líneas de investigación de sus programas y proyectos (especialmente relacionado con Ingeniería de Computación, Ingeniería Civil, Ingeniería Electromecánica, Ingeniería Industrial, Arquitectura y Urbanismo, Derecho, Relaciones internacionales, Psicología, Administración Pública, Gerencia y Mercadeo, Finanzas y Contabilidad.

${ }^{5}$ Puede acceder mediante el enlace: https://pkp.sfu.ca/about/organization/ 
Inicialmente se publicó en formato físico, en el mes de junio de 2015, con diez artículos de investigación, uno en español, uno en francés y ocho en portugués, que abarcaban las diversas áreas de conocimiento. Sin embargo, en el año 2016 la revista se encontraba en el dilema de seguir publicando en físico o acceder a la plataforma virtual en la búsqueda de la internacionalización de sus procesos de investigación.

En este sentido, en enero de 2017 se decide dar apertura a la publicación digital, tomando en cuenta sus grandes ventajas, dando inicio al proyecto de implementación del OJS y actualización de la revista Sapientiae en el mismo. Para lo cual, el primer procedimiento requerido fue la obtención del ISSN en formato digital, ya que poseía solo la aprobación del ISSN para el formato físico (2183-5063). Luego de este paso, se inició el proceso de personalización del OJS en el servidor de la UÓR.

El desarrollo del proyecto con la revista Sapientiae en la UÓR se llevó a cabo en dos fases, la primera, referida a la instalación del software OJS, en el mes de marzo de 2017, para lo cual la UÓR adquirió un nuevo servidor para garantizar el respaldo de la información de la revista Sapientiae.

Posteriormente el software fue alojado en el servidor y actualmente se encuentra disponible en el enlace http://publicacoes.uor.ed.ao/index.php/ sapientiae/ como se observa en la Figura 1.

FIGURA 1

Visualización de la revista Sapientiae en la web

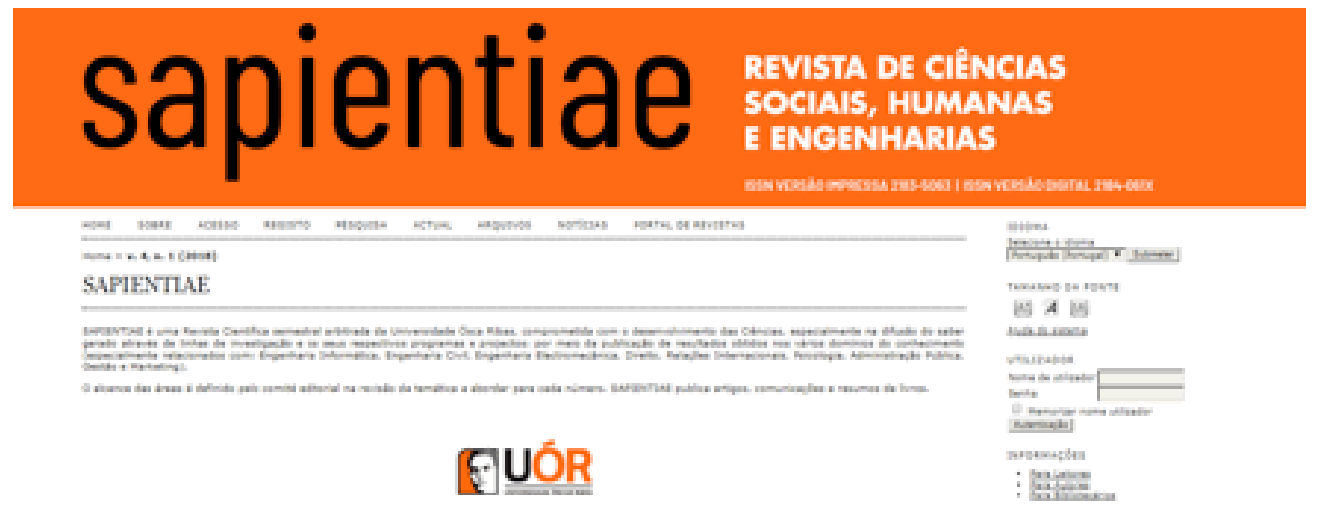

Fuente: Sapientiae $(2019)^{6}$

Esta primera fase incluyó la instalación y personalización del sitio, así como el alojamiento del contenido de la revista Sapientiae ya publicado en formato físico, contando así con la revista en versión electrónica y física.

Desde mayo hasta octubre de 2017 se procedió a actualizar los números de la revista, culminando con el más reciente numero 4-1 (Julio-Diciembre 2018). Actualmente, los volúmenes mencionados se pueden acceder sin restricciones desde cualquier parte del mundo, en formato pdf.

La segunda fase estuvo referida al interés de la dirección de la UÓR en la capacitación de su personal docente y de investigación en el manejo de 
la plataforma OJS. Esta fase se llevó a cabo en el mes de julio de 2017, a través de cursos didácticos y científicos, además de asesoría personalizada en el manejo del OJS en las instalaciones de la UÓR. En el mismo, se tuvo la oportunidad de conocer el sistema, sus funcionalidades y los distintos roles y funciones que podían cumplir a través de este. Dicho adiestramiento incluyó la práctica en el sistema, lo cual facilitó a los participantes la comprensión del mismo.

Esta capacitación sirve de soporte a los participantes en el desarrollo de la carrera investigativa, ya que, en el ámbito científico, las instituciones educativas se han interesado por la implementación de este sistema en la búsqueda de la estandarización de los procesos y la calidad de los mismos, garantizando transparencia y objetividad en el manejo de documentos que se presentan en el ámbito científico y que constituyen aportes a las distintas áreas de la ciencia.

En este sentido, la revista Sapientiae se suma a los casos de éxito de cómo el montaje de una revista en un sistema de acceso abierto puede traer incontables beneficios a la institución, los investigadores y la ciencia de cualquier país; destacando la evolución en la indización de la revista Sapientiae en las bases de datos señaladas en la tabla 4.

TABLA 4

Índices y fuentes secundarias de indización

\begin{tabular}{lccc}
\hline \multicolumn{1}{c}{ ÍNDICE } & SOLICITUD & ACEPTACIÓN & PAís \\
\hline $\begin{array}{l}\text { Actualidad } \\
\text { Iberoamericana }\end{array}$ & Oct-17 & Nov-17 & Chile \\
I2OR & Oct-17 & Nov-17 & India y Australia \\
DIALNET & Oct-17 & Dic-17 & España \\
REDIB & Oct-17 & Dic-17 & España \\
Google Scholar & Ingresado en Octubre 2017 & Mundial \\
Sherpa Romeo & Oct-17 & En espera & U.K. \\
IRESIE & Oct-17 & En espera & México \\
MIAR & Dic-17 & Feb-17 & España \\
OAJI.net & Dic-17 & Mar-18 & Rusia \\
DOAJ & Feb-17 & May-18 & Mundial \\
CITEFACTOR & Mar-18 & Mar-18 & USA/Canadá \\
Clarivate Analytics & Mar-18 & En espera & USA \\
Scielo & Mar-18 & En espera & Mundial \\
CORE & Jun-18 & Ago-19 & U.K. \\
Scientific $\quad$ Indexing & Abr-18 & Jun-18 & USA \\
Services & & & Mgo-18 \\
DEYCRIT-Sur & Ago-18 & Ago &
\end{tabular}




\begin{tabular}{lccc}
\multicolumn{1}{c}{ ÍNDICE } & SOLICITUD & ACEPTACIÓN & PAís \\
Science library index & Ago-18 & Ago-18 & India \\
& & & \\
Livre & Ago-18 & Sep-18 & Brasil \\
ERIHPLUS & Sep-18 & Oct-18 & Noruega \\
LATINRED & Dic-18 & Dic-18 & Argentina \\
ROAD & Registrado por ISSN internacional & Mundial \\
JOURNALS FOR FREE & Oct-18 & Ene-19 & Portugal \\
& & & \\
\hline
\end{tabular}

Fuente: elaboración propia.

4. Retos y perspectivas de desarrollo científico y tecnológico del Ministerio de Educación Superior, Ciencia, Tecnología e Innovación de Angola (MESCTI)

Entre las bases de datos donde Sapientiae es la única revista de Angola a lograr su indización, se destaca la DOAJ, DIALNET y MIAR. Como es reconocido por la comunidad científica nacional, estar incluida en los índices internacionales da la posibilidad de que más investigadores puedan tener acceso a la revista Sapientiae, aspecto que garantiza su conocimiento en el ámbito internacional y el desarrollo de la ciencia en Angola.

El trabajo de mejora de la revista Sapientiae es continuo, y busca el desarrollo de elementos apegados a los estándares internacionales que manejan las revistas mejor posicionadas, y tener la oportunidad de ingresar a las mismas en el mediano y largo plazo. Actualmente el Comité Editorial de la revista Sapientiae trabaja en el desarrollo de las indicaciones éticas y buenas prácticas, manejando la posibilidad de suscribirse al Comité de Ética para Publicaciones (COPE, 2019) ${ }^{7}$.

El tema de la ética en las publicaciones ha sido estudiado en los últimos años por distintos investigadores (Díaz-Campo y Segado-Boj, 2016), quienes señalan que un $11,2 \%$ de las revistas que no incluían el tema ético en sus normas y aquellas que lo incluyen se centran en el tema de la duplicación de información y el plagio, dejando de lado elementos como las responsabilidades de los editores y las sanciones.

La insuficiente articulación de los factores que dinamizan el proceso de investigación y de producción científica de calidad limitan actualmente la visibilidad internacional de resultados producidos por investigadores angolanos (Ministerio de Ciencia y Tecnologia, 2013; Gungula Wongo, Castillero Velasquez y Mendes da Conceição Barbosa Rodrigues, 2017), así como la dispersión de iniciativas y esfuerzos para el desarrollo de buenas prácticas de extensión universitaria y el escaso estímulo para el desarrollo de una cultura de circulación de información científica, técnica, pedagógica y administrativa. No obstante, se destacan las potencialidades de los investigadores angolanos, comprometidos con el mejoramiento de los indicadores para cambiar cualitativamente el actual escenario. 
Según Guambe y Bueno de la Fuente (2013), la producción científica de Angola ocupó del 2002 al 2012 el $18 \%$ de la producción científica de los países de habla lusofona en África, con 380 documentos publicados según la base de datos Scopus. Es importante destacar que estos datos son mayormente conocidos por investigadores internacionales que poseen acceso a estas bases de datos, debido a que por el control de cambio monetario actualmente ninguna universidad puede subscribirse a las mismas, seguramente, para ampliar la visión habría que buscar información hacia documentos publicados de forma distinta, pero sería tema de otro artículo.

En aras de reactivar el compromiso de implementación efectiva del Sistema Nacional de Ciencia, Tecnología e Innovación, en diciembre del 2017 se realizó una exploración nacional promovida por el MESCTI, la cual contó con la participación de más de treinta IES públicas y privadas. Durante dicho proceso, el MESCTI creó las condiciones objetivas para trazar una posible imagen que caracterizara la gestión actual de las IES, tanto en relación con la mejoría de la calidad del proceso docente-educativo, como de las investigaciones científicas desarrolladas en las distintas IES.

En dicha exploración se constató el aumento discreto de actividad científica con publicaciones en revistas indexadas e internacionalmente reconocidas, así como la participación de docentes angolanos en eventos científicos nacionales e internacionales. Sin embargo, se evidencia la necesidad de mejorar la calidad de los proyectos presentados, poner a disposición recursos desde el MESCTI para estimular la realización de investigación científica; la creación de redes nacionales de investigadores, por área de conocimiento, visando el fortalecimiento de protocolo interinstitucional; la publicación de resultados de los proyectos de investigación, entre otros (Ministerio de Educación Superior, Ciencia, Tecnología e Innovación, 2017).

Además de lo expuesto, el MESCTI reveló en marzo del 2018 que está estableciendo contactos para fortalecer la cooperación con distintas instituciones internacionales, donde a modo de ejemplo se menciona la Academia de Ciencias de África, con vista a crear instrumentos que posibiliten estimular la creación de sistemas abiertos de publicación científica.

Se destaca, además, la necesidad de concertar alianzas estratégicas que contribuyan al desarrollo y al avance del sector de las Tecnologías de Información y Comunicación (TIC) en Angola, un incentivo al uso y desarrollo de tecnologías de acceso abierto, así como la creación de redes de investigadores y de espacios para la promoción de proyectos. Desde esta perspectiva, el Plan de Desarrollo Nacional 2018-2022 (Ministerio de Economía y Planeación, 2018) proyecta la promoción de la Innovación y Transferencia de Tecnología (en su programa 2.2.4), retos que tienen que ver con la creación de Polos Tecnológicos y de incubadoras de empresas de base tecnológica.

En ese contexto, el interés en perfeccionar el proceso de publicaciones científicas es cada vez más notable en las distintas instituciones de educación superior. Prueba de ello fue la organización de una sesión de formación introductoria que se llevó a cabo en la provincia de Namibe, Angola, del 26 al 27 de abril del 2018, en donde se recogieron impresiones de los 24 profesores asistentes de la Escuela Superior Pedagógica de Namibe, del Instituto Superior de Ciencias de Educación de Huíla, de la Academia de Pescas de Namibe y de la Universidad Óscar Ribas de Luanda. 
El referido interés está consustanciado en la creación de revistas científicas, el desarrollo consciente de proyectos de investigación científica, la comprensión de la importancia de este tema en la actualidad, además de la motivación de cada institución participante en implementar experiencias similares que viabilicen la publicación y el aumento de sus estándares en investigación.

Entre las sugerencias recogidas en dicha sesión formativa, se destacan las relacionadas con las diligencias necesarias para que el MESCTI estimule la implementación de las revistas científicas en las distintas instituciones de educación superior, la creación de condiciones para que regularmente se capaciten los docentes en temáticas inherentes a la redacción científica, además de desarrollar una política para incentivar la publicación de artículos como perfeccionamiento de la carrera investigativa.

En ese proceso no se puede dejar de lado la Recomendación sobre la Ciencia y los Investigadores Científicos hecha por la Organización de las Naciones Unidas para la Educación, la Ciencia y la Cultura (UNESCO, 2017, p. 156) en donde resalta que

Los Estados Miembros deberían alentar y facilitar la publicación de los resultados obtenidos por los investigadores científicos y ampliar esta práctica a los datos, métodos y programas informáticos utilizados, a fin de ayudarlos a compartir la información científica y a adquirir la reputación que merezcan, así como de promover las ciencias, la educación y la cultura en general.

Al mismo tiempo alientan el acceso abierto, siendo el Open Journal System (OJS) una forma para el logro de este fin. En este sentido, el MESCTI de Angola se encuentra alineándose a los requisitos de la comunidad internacional al promover y ampliar la cultura de investigación científica en todas las Instituciones de Educación Superior.

\section{Consideraciones finales}

La elaboración de la presente investigación tiene un carácter inédito al presentar resultados contextualizados a la realidad angolana sobre los procesos de creación y gestión de revistas científicas en general y, de modo particular, en la provincia de Luanda, aspecto que clarifica la insuficiente experiencia sistematizada por las distintas Instituciones de Educación Superior.

Los resultados obtenidos permiten la identificación de necesidades prioritarias que, de ser solucionadas, pueden contribuir al desarrollo de competencias de redacción de artículos científicos, gestión de revistas científicas e innovación tecnológica, como alternativa para la internacionalización de la labor desarrollada en las distintas Instituciones de Educación Superior en la provincia de Luanda.

A nivel de la referida provincia existen menos de diez revistas científicas arbitradas e indizadas en bases de datos de prestigio internacional, aspecto que poneen duda el compromiso de los gestores de Instituciones de Educación Superior. Esto demuestra la posibilidad de creación y mantenimiento de una revista científica de acceso abierto desde una Institución de Educación 
Superior angolana, usando el Open Journal System como herramienta para la difusión del conocimiento, así como el avance que se puede tener en el corto y mediano plazo en la internacionalización de la misma, tomando las decisiones adecuadas. Dicha experiencia puede servir como ejemplo para el resto de las instituciones en el desarrollo de los nuevos retos y perspectivas del Ministerio de Educación Superior, Ciencia, Tecnología e Innovación para el desarrollo de la ciencia en Angola.

A pesar del limitado enfoque en la necesidad de implementación de sistemas abiertos de divulgación científica en Angola, la perspectiva nacional de incluir dos Instituciones de Educación Superior entre las cien mejores Universidades Africanas en el ranking académico hasta el año 2022, es indicio de que el Ministerio de Educación Superior, Ciencia, Tecnología e Innovación de Angola, deberá accionar todos los mecanismos técnicos y científicos que posibiliten este recto.

La experiencia de instalación e implementación del Open Journal System para la revista Sapientiae de la Universidad Oscar Ribas en Luanda-Angola es una muestra del uso del sistema desde cero en una Institución de Educación Superior, que se puede tomar como prueba piloto para la implementación del sistema en cualquier otra institución.

\section{Referencias}

Agencia Angola Press. (2018). Angola recoge indicadores sobre Ciencia y Tecnología. Angola Press. Recuperado de http://www.angop.ao/angola/ es_es/noticias/ciencia-e-tecnologia/2018/6/28/Angola-recoge-indicadoressobre-Ciencia-Tecnologia,99dfed20-47af-468c-82fb-adef04e7ae41.html

Angola Formativa. (2018). Catalogo Angolano de Cursos superiores. Lista de Instituciones de Enseñanza de Angola. Recuperado de https://www. angolaformativa.com/pt/centros/

Borrero, D. (10 de noviembre de 2017) Angola: un país que se reconstruye con éxito. Granma. Recuperado de http://www.granma.cu/ mundo/2017-11-10/angola-un-pais-que-se-reconstruye-conexito-10-11-2017-22-11-13

Claudio-González, M.G. y Villarroya, A. (2015). Desafíos de la edición de revistas científicas en acceso abierto. El Profesional de la Información, 24(5), 517-525. Doi: http://dx.doi.org/10.3145/epi.2015.sep.02

Comité de Ética para Publicaciones. (2019). Promoting integrity in research and its publication. Recuperado de https://publicationethics.org/

Díaz-Campo, J., y Segado-Boj, F. (2016). Indicaciones Éticas en las revistas de educación indexadas en la WOS. El Profesional de la Información, 25(5), 738-746. Doi: https://doi.org/10.3145/epi.2016.sep.04

Faustino, A., Pérez Sánchez, N. y Gungula Wongo, E. (2017). Formación del Pensamiento Complejo Ingenieril en la Universidad Óscar Ribas. TELOS. Revista de Estudios Interdisciplinarios en Ciencias Sociales, 19(3), 523-544. Recuperado de https://dialnet.unirioja.es/servlet/ articulo?codigo $=6219245$ 
Guambe, Martins, F. y Bueno de la Fuente, G. (2013). Disponibilidade em Acesso Aberto da produção científica da África Lusófona. INCID: Revista de Ciência da Informação e Documentação, 4(2), 5-19. Recuperado de http://www.revistas.usp.br/incid/article/view/69267

Gungula Wongo, E., Castillero Velasquez, J. y Mendes da Conceição Barbosa Rodrigues, M. (2017). Visibilidade dos Sistemas de Créditos Académicos nos Países Lusófonos. TELOS. Revista de Estudios Interdisciplinarios en Ciencias Sociales, 19(2), 288-307. Recuperado de https://dialnet. unirioja.es/servlet/articulo?codigo $=6219234$

Hernández Pina, F. y Maquilón Sánchez, J. (2010). Indicadores de calidad de las revistas científicas y sistema de gestión editorial mediante OJS. Revista De Investigación Educativa, 28(1), 13-29. Recuperado de http:// revistas.um.es/rie/article/view/109941

Ministerio de Ciencia y Tecnologia. (2013). Documentos Reitores da Ciência, Tecnologia e Inovação em Angola. Angola: Autor

Ministerio de Educación Superior, Ciencia, Tecnología e Innovación. (2017). Síntese da Auscultação às Instituições de Ensino Superior Angolanas, Públicas e Privadas. Luanda, Angola.

Organización de las Naciones Unidas para la Educación, la Ciencia y la Cultura. (2017). Recomendación sobre la Ciencia y los Investigadores Científicos. Recuperado de http://ciencia.ao/images/noticias/Docs/ UNESCO_RecommendationResearchers_Nov2017_ES.pdf

Ministerio de Economía y Planeación. (2018). Plan de Desarrollo Nacional 2018-2022. Angola. Recuperado de https://www.info-angola. com/attachments/article/4867/PDN\%202018-2022_MASTER_vf_ Volume\%201_13052018.pdf

Public Knowledge Project. (2017). Recursos OJS en español. Recuperado de https://pkp.sfu.ca/recursos-ojs-en-espanol/

Public Knowledge Project. (2019). Organization. Recuperado de: https:// pkp.sfu.ca/about/organization/

Régimen Jurídico de Evaluación y Acreditación de Calidad de las Instituciones de Educación Superior. (2018). Decreto Presidencial n. ${ }^{\circ}$ 203/18, de 30 de agosto. Luanda, Angola: Imprenta Nacional.

Sapientiae (2019). Visual de la revista. Recuperado de http://publicacoes. uor.ed.ao/index.php/sapientiae/

Scientific Electronic Library Online (Scielo). (9 de enero de 2014). A bibliometria do mundo em desenvolvimento. SciELO em Perspectiva. [mensaje en un blog]. Recuperado de http://blog.scielo.org/ blog/2014/01/09/a-bibliometria-do-mundo-em-desenvolvimentopublicado-originalmente-na-newsletter-da-elsevier-research-trendsissue-35-developing-research-in-developing-countries/ 
Willinsky, J., Stranack, K., Smecher, A., Macgregor, J. y Acevedo, A. (2010). Open Journal Systems: Una guía completa para la edición de publicaciones en línea. PKP. Recuperado de http://pkp.sfu.ca/ojs/docs/ userguide/2.3.3/es/index.html 


\section{e-Ciencias de la Información}

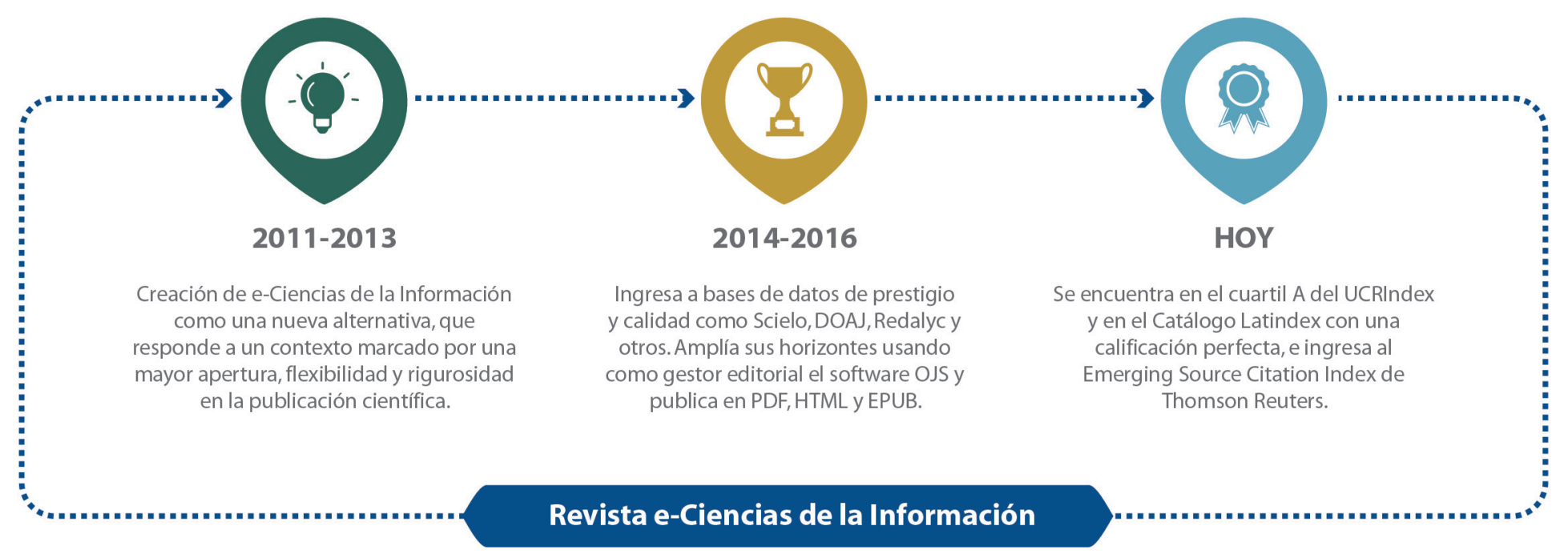

¿Dónde se encuentra idexada e-Ciencias de la Información?

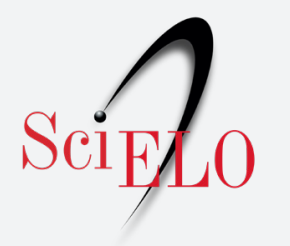

\section{latindex 12 clarivate Analytics}

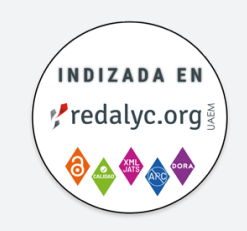

Para más información ingrese a nuestra lista completa de indexadores

¿Desea publicar su trabajo?

Ingrese aquí

O escríbanos a la siguiente dirección revista.ebci@ucr.ac.cr 